\title{
Prosthetic Rehabilitation of Rhino Orbital Mucormycosis Associated with COVID-19: A Case Series
}

\author{
MB Ravi (D) \\ Sowmya Srinivas iD \\ Ekaterina Silina (iD ${ }^{2}$ \\ Soumee Sengupta (D) \\ Tanvi Tekwani id \\ Raghu Ram Achar (iD ${ }^{3}$ \\ 'Department of Prosthodontics, JSS \\ Dental College and Hospital, JSS \\ Academy of Higher Education \& \\ Research, Mysuru, Karnataka, India; \\ ${ }^{2}$ Department of Human Pathology, I.M. \\ Sechenov First Moscow State Medical \\ University (Sechenov University), \\ Moscow, Russia; ${ }^{3}$ Division of \\ Biochemistry, School of Life Sciences, JSS \\ Academy of Higher Education \& \\ Research, Mysuru, Karnataka, India
}

\begin{abstract}
Mucormycosis, a rare fungal infection seen in diabetes, is now very frequent owing to the deadly triad of COVID-19 infection, diabetes, and rampant use of corticosteroids. Immediate management revolves around therapeutic drugs like antifungals, antibiotics, and aggressive surgical debridement. The cases described in the article explain prosthetic rehabilitation of maxillectomy defects. The findings focus on prosthetic rehabilitation of patients with acquired maxillectomy defects after mucormycotic necrosis post-COVID-19 infection and the techniques to overcome the complications like lack of supporting tissues and post-surgical microstomia. The maxillectomies were performed on patients who suffered a superinfection of mucormycosis after COVID-19 contraction and uncontrolled blood sugar levels. Case 1 elaborates a technique to overcome the complications like lack of supporting structures and microstomia by fabrication of sectional and hollow obturator prostheses using sectional impression technique and lost salt technique. Case 2 explains the management of an extensive defect with a mobile soft tissue flap and lone standing tooth by using a functional impression technique to gain retention and support from the remaining soft and hard tissues. Both the techniques overcome the clinical complications and give predictable outcomes. Prosthetic rehabilitation of such challenging cases needs modifications depending upon the clinical challenges encountered.
\end{abstract}

Keywords: maxillectomy, microstomia, sectional impression, lost salt technique, functional impression

\section{Introduction}

Severe acute respiratory syndrome coronavirus 2 (SARS-CoV2), spread from Wuhan, China, has been declared the 6th Public Health Emergency of International Concern (PHEIC). ${ }^{1}$ Since the outbreak of COVID-19, with every mutation, it has posed various challenges to human life and the field of health sciences. It is known that coinfection and superinfection are common in any viral infection. Coinfection occurs simultaneously, whereas superinfection develops following the initial infection. The occurrence of the same is quite common with COVID-19 and it increases with an idyllic environment of decrease in oxygen saturation level in the body. Systemic conditions like high glucose levels, diabetic ketoacidosis, and increased steroid therapy are also common factors for developing superinfection. Immunocompromised patients have a higher chance of developing infections from bacteria, viruses, and fungi due to a lack of basic cellular mechanisms. ${ }^{2}$
Correspondence: MB Ravi Department of Prosthodontics, JSS Dental College and Hospital, JSS Academy of Higher Education \& Research, Shri Shivarathreeshwara Nagar, Mysuru, Karnataka, 5700I5, India

Email dr.ravimb@jssuni.edu.in 
Among fungal infections, Mucormycosis is the third most common opportunistic infection recorded after candidiasis and aspergillosis. ${ }^{3}$ According to WHO, Mucormycosis is a non-contagious yet aggressive and life-threatening infection. Patients on corticosteroids or with comorbidities like diabetes mellitus are at increased risk of post-COVID infections like Mucormycosis. ${ }^{4-6}$ Globally, the mortality rate of Mucormycosis associated with diabetes is about $46 \%{ }^{7}$ According to Prakash et al, ROCM (Rhino-Orbito-Cerebral Mucormycosis) is the most common clinical form of Mucormycosis in India $^{3}$ with $88 \%$ being associated with diabetes mellitus. ${ }^{8}$ Mucormycosis requires immediate attention and delay leads to increased mortality. ${ }^{9,10}$

The first line of treatment for ROCM is Liposomal amphotericin B, second line/step down therapy is Posaconazole, Isavuconazole, and aggressive surgical debridement of the necrotic tissue upon confirmation of this infection. ${ }^{11,12}$ Aggressive surgical debridement like maxillectomy alters facial appearance, functions, and an individual's overall quality of life. ${ }^{13}$ Maxillectomy can be reconstructed with soft tissue flaps or a prosthetic obturator. ${ }^{14}$ Obturator function and quality of life have a strong correlation.

Some of the problems associated with rehabilitating extensive intraoral defects make it extremely challenging for a prosthodontist to fabricate a prosthesis. ${ }^{15}$ Here, we report on prosthetic rehabilitation of maxillectomy patients post mucormycotic necrosis, from recording an accurate impression of the defect until the construction of the prosthesis. The expected outcome is an improvement in the overall function and aesthetics after the delivery of prostheses.

\section{Case History \\ Case I}

A 34-year-old female patient reported for prosthetic rehabilitation of an acquired defect after surgical debridement of mucormycotic necrosis. The illness started as a simple toothache about 6 weeks ago and was advised for extraction. Further dialogue history revealed that the patient had taken only one vaccination dosage against the COVID-19 disease. Investigations revealed FBS of $273 \mathrm{mg} / \mathrm{dl}$, PPS of $295 \mathrm{mg} /$ $\mathrm{dl}, \mathrm{HbA} 1 \mathrm{c}$ of $10.1 \%$, and a C-reactive protein level of $0.98 \mathrm{mg} / \mathrm{dl}$, and the extraction was postponed. After visiting multiple dentists and a district hospital, a $\mathrm{KOH}$ Mount of her tissue confirmed the presence of aseptate hyphae and culture yielded Rhizopus. The right maxillectomy biopsy specimen also suggested features of Mucormycosis thus a surgical debridement and resection was carried out. The patient was administered with piperacillin and tazobactam $4.5 \mathrm{gm}$ IV B. I.D., Inj. Metrogyl 100 mL T.I.D., Inj. Posaconazole $300 \mathrm{~mL}$ Q.D., Inj. Insulin $1000 \mathrm{~mL}$ (basalog) as pre-operative and post-operative medications with nutrition supplements of $\mathrm{D}$ protein powder and potchlor syrup and was intubated until prosthetic rehabilitation.

A general examination revealed that the patient presented with an inability to walk and a malnourished build. On extraoral examination, it was noted that the patient had a fair complexion with a gross asymmetry on the right side of the mid-facial region. Scarring on the philtrum and under the right eye; with a collapsed mid-face was observed. Mouth opening was measured to be approximately 15 to $17 \mathrm{~mm}$ only (Figure 1).
A

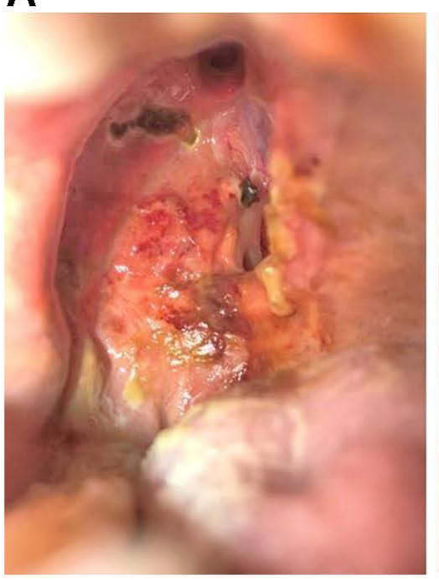

B

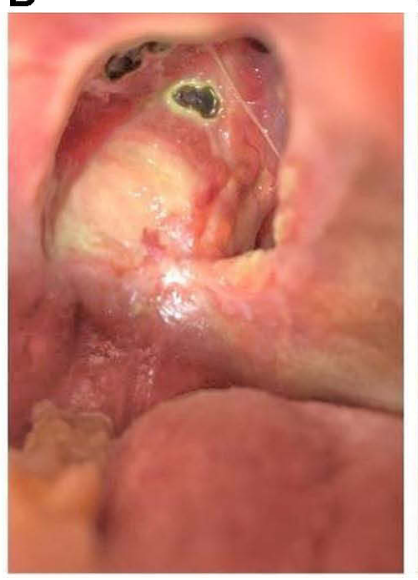

C

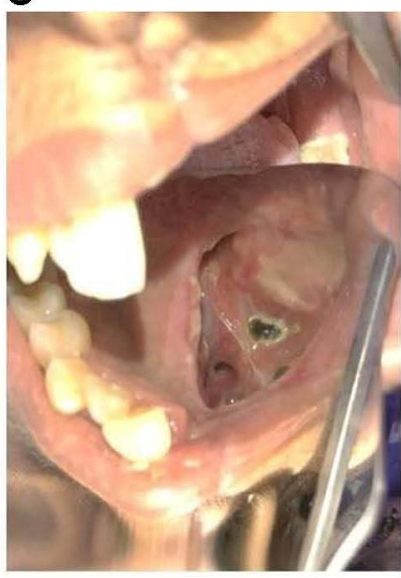

Figure I Intraoral photographs depicting the extent of the lesion. (A) Intraoral defect on the first visit showing necrosis and sloughing on the margins of the defect. (B and C) Intraoral photographs of the defect taken after I month of the prosthesis delivery. 
An intraoral examination revealed a defect falling in Aramany's class 1 and total maxillectomy with orbital contents intact. The entire hard, soft palate and teeth were resected on the right side. ${ }^{16}$ Mild tissue inflammation was noted at the margins of the defect. The teeth missing were 11 to 18,31 , and 41 . The residual teeth present for retention and support were 21 to 28,32 to 38 and 42 to 48 . Crowding and generalized recession were noted concerning these teeth. Multiple posterior supra-erupted teeth were present, thus, making insertion of tray and prosthesis further difficult.

The patient complained of difficulty in mastication, speech, and deglutition. The prosthetic challenge posed here was the absence of any alveolar bone on the right side to gain retention and support, from the post-operative complication of microstomia. A preliminary impression was made with irreversible hydrocolloid and poured with type III gypsum product (dental stone, Kalabhai). Due to the reduced mouth opening, it was decided that the second impression would be taken in sections.

The special tray was fabricated in sections using detachable buttons to record the secondary impression of the maxillary defect with an accurate seal (Figure 2A). The first part recorded was of the defect using an impression compound supported with finger pressure. Adequate precautions were taken to not let the impression compound completely set in the mouth to easily remove it directly with the hand. After the compound was removed from the oral cavity, light body elastomeric impression material was injected over it, and a wash impression was made (Figure 2B). This step ensured a complete sealing of the defect. The undersurface of the compound was now heated, and threads were attached in a cruciform manner for the reorientation of the sectional impression outside the oral cavity (Figure 2C). The impression compound with the threads in place was placed back in the mouth. ${ }^{17}$ The first part of the sectional tray was inserted into the mouth to check for any interference with the teeth or impression compound. Now, this sectional tray was inserted in the first quadrant, an impression using putty consistency of additional silicones was made and allowed to set intraorally. The second part of the sectional impression was made of the second quadrant using irreversible hydrocolloid impression material and allowed to set. The impression was removed in 3 parts, they being the two sections individually and the impression compound; which were then approximated outside the oral cavity (Figure 2D).

The secondary impression was then poured into the type III gypsum product. Jaw relation was done followed by mounting on a mean value articulator. The trial of the denture was carried out routinely. The trial prosthesis was evaluated for lip fullness, speech, and vertical dimension. The denture was fabricated in sections and made hollow to reduce the weight for the ease of insertion for the patient (Figure 3). Denture sections were attached with the use of Neodymium-iron-boron intraoral magnets (Figure 4). The final prosthesis carefully evaluated was interference, finished and polished. Nutritional counseling, post-insertion, and cleaning instructions were given to the patient (Figure 5).

\section{Case 2}

A 60-year-old male farmer was referred for the fabrication of an interim obturator for closure of his palatal defect after undergoing surgery for Mucormycosis of the upper jaw bone and associated structures. History revealed that the patient had first experienced dry cough, body ache, and generalized fatigue two months ago. Four days after the initial onset, he
A

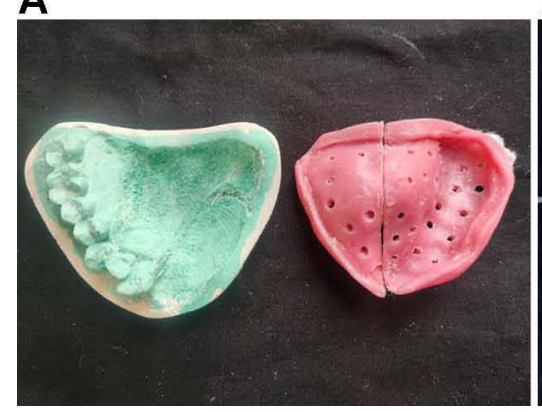

B

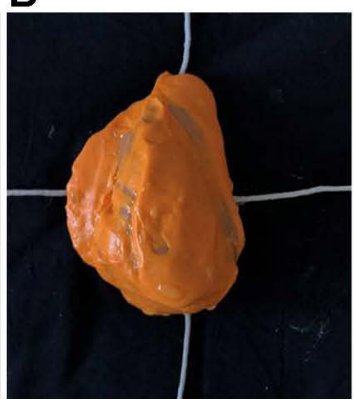

C

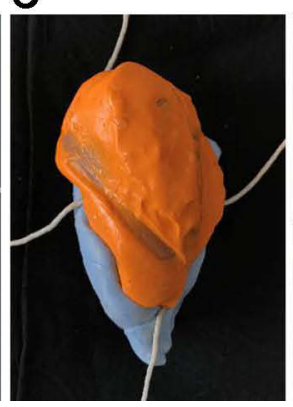

D

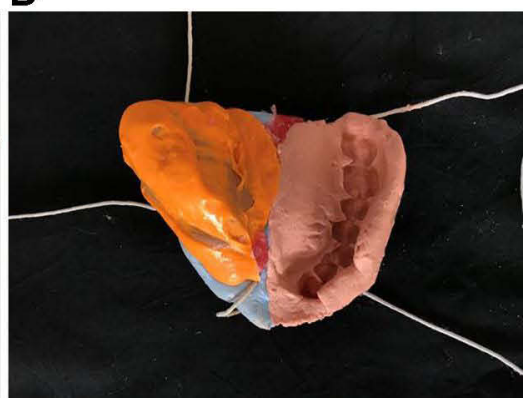

Figure 2 Impression making for the obturator prosthesis. (A) Fabrication of perforated custom-made sectional impression tray on the previously obtained preliminary cast. (B) First part of the impression of defect made with impression compound and wash impression made with light body elastomeric impression material. (C) Second part of the impression made with sectional custom tray, with elastomeric impression material of putty consistency as a pick up impression of the defect side. (D) Third part of impression of contralateral side made with irreversible hydrocolloid impression material, followed by removal of the three part impression in sections and reapproximated outside the oral cavity. 


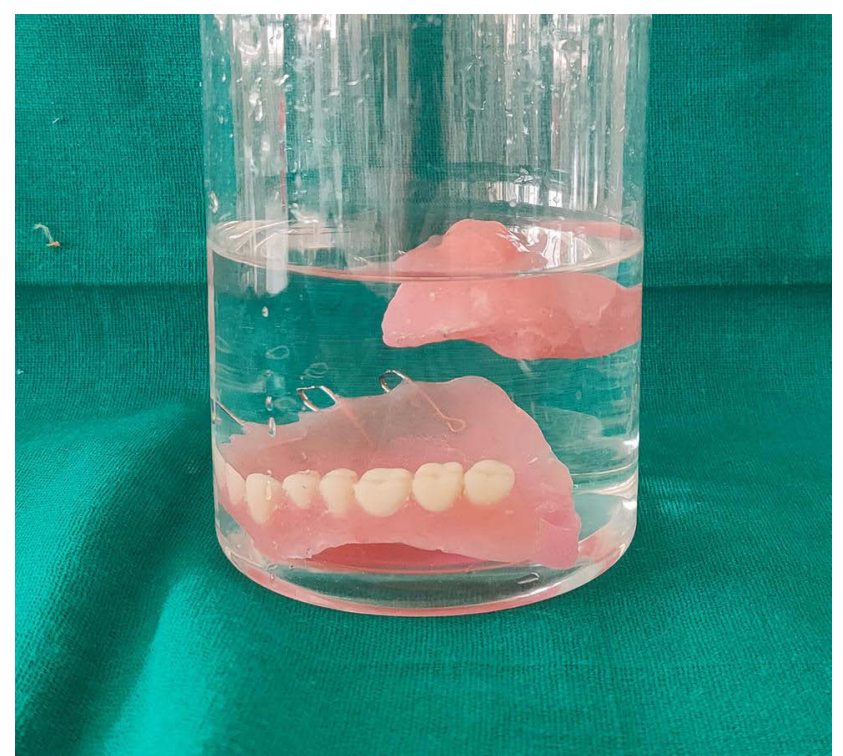

Figure 3 Hollow sectional prosthesis, where antral part made hollow by lost salt technique and confirmed with positive water test and illustrated by the floating of the antral bulb.

developed a toothache in the upper jaw that was diffuse, dull, continuous and gradually progressive. The severity increased within two days, radiating to the entire right side of his face with no apparent aggravating or relieving factors. By this time, he noticed multiple intraoral ulcerations with diffuse inflammation in his gums and mild swelling over the right side of the face and palate. Patient experienced no relief upon taking the prescribed antibiotics and painkillers. In order to rule out diabetes, a screening analysis of random plasma glucose was performed to reveal elevated RBS of $211 \mathrm{mg} / \mathrm{dl}$. No COVID19 vaccine doses were administered to the patient.

Investigation included was diagnostic anterior rhinoscopy, which depicted blackish discoloration over bilateral nasal turbinates. Direct $\mathrm{KOH}$ mounting and histopathological frozen sections of affected palatal tissue revealed broad aseptate hyphae of Mucorales. Pus noted anterior to the
Eustachian tube opening and from over the right alveolus, when sent for gram staining and culture sensitivity tests, confirmed the presence of Klebsiella pneumoniae and Streptococcus spp. Radio imaging included Gadoliniumenhanced MRI of the brain with orbits and Paranasal sinus (PNS) and a plain CT of PNS, both of which showed features of right maxillary invasive fungal sinusitis with maxillary bone osteomyelitis (Figure 6). The patient was diagnosed with Right Rhinosinomaxillary Mucormycosis with Left Mucormycosis. Surgically, management was done with the right Total Maxillectomy and left Hemimaxillectomy. Post-surgically, the patient was administered with Inj. Posaconazole and Inj. Amphotericin after obtaining nephrological clearance for the latter. Patient was advised to take nutritional supplements like prohance D Powder and syrup Potklor. Intraorally, a persistent, extensive palatal gaping had been noted, for which, the patient had been referred for the fabrication of the obturator.

On extraoral examination, no gross asymmetry was noted. No abnormality was noted in the TMJ movements and lymph nodes. There was an adequate mouth opening. Intraoral examination revealed an extremely deep communication between the oral and nasal cavities, which fell into the Aramany Class IV category, in the hard and soft palate. ${ }^{18}$ There was a total lack of hard and soft palate concerning the missing teeth, ie 11 to 18 on the right side and 21 to 26 on the left side. The only tooth present in the upper arch was a grade I mobile 27 with limited alveolar support. A U-shaped movable mucosal flap was seen in the posterior $1 / 3^{\text {rd }}$. of the hard palate. Diffuse erythematous areas with discrete areas of crustations and sloughing were noted on the adjacent oral mucosa (Figure 7). In the lower arch, there was only 36 missing with an existent RPD, fabricated 1 year back.

The devised prosthetic treatment plan consisted of a transitional prosthesis to close the defect, taking retention and support from anatomic undercuts within the
A

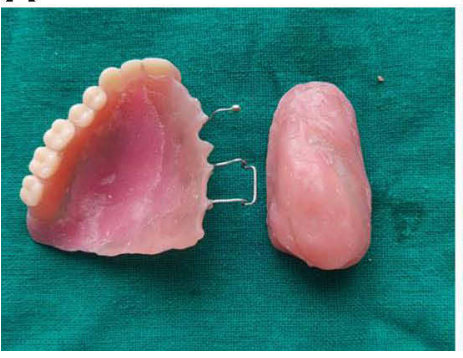

B

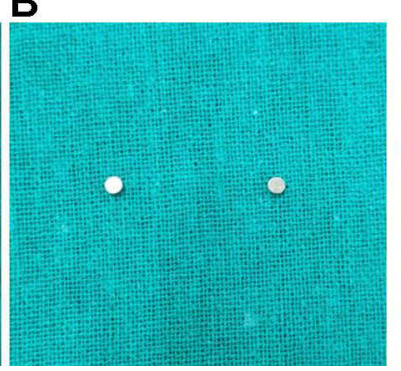

C

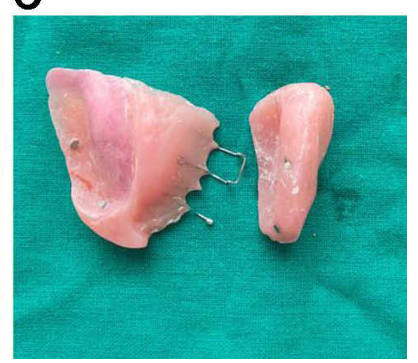

Figure 4 Fabrication of denture in sections and their approximation with magnets. (A) Polished surfaces of the sectional denture. (B) Neodymium-Iron-Boron rare earth element rust free magnets with a diameter of $3 \mathrm{~mm}$ and thickness of $1.5 \mathrm{~mm}$. (C) Intaglio surfaces of the sectional denture. 


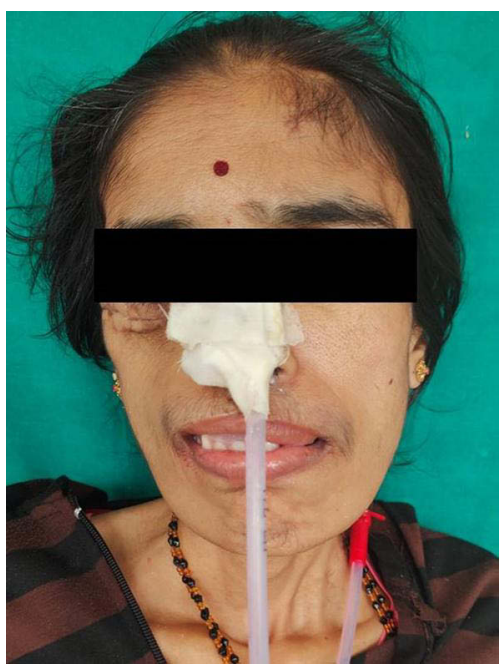

Figure 5 Post-operative extraoral view of the patient after the insertion of the denture.

defect, direct retention from the lone standing 27 , and to be made hollow to reduce the total weight.

After taking necessary precautions, maxillary and mandibular preliminary impressions were made with an irreversible hydrocolloid (DPI Algitex, India) and poured with Type 2 Dental plaster and Type 3 Dental stone, respectively (Kaldent, Kalabhai, India). A special tray was fabricated for the upper arch with an auto-polymerizing acrylic resin and a second primary impression again made with thickconsistency alginate and the second cast poured with Type 3 Dental stone (Kalstone, Kalabhai, India). A heat-cured transitional plate was fabricated, and its intaglio surface was generously coated with an acrylic tissue conditioner (GC Corporation, Tokyo, Japan) to make a functional impression of the defect, and the patient was advised to wear it in his natural environment for the next 24 hours. After the 24-hour recall, a pickup impression was made and poured with Type 3 Dental stone (Figure 8)

Adequate lip fullness, visibility, and a proper vertical dimension were established; and centric relation was recorded. The teeth arrangement was done on a mean value articulator with anterior semi-anatomic and posterior non-anatomic teeth (Premadent, National Dental Supply, India). After trial insertion, the denture was processed (Trevalon HI Denture Base Material, Dentsply, India) and the antral bulb was made hollow by the lost salt technique. Denture insertion was done and checked for occlusion, phonetics, and aesthetics (Figure 9).

Post-insertion adjustments were made after 24,48 , and 72 hours of denture insertion. Proper instructions were given about denture hygiene, placement, and removal. Nutrition counseling was done, keeping in mind, his systemic health and the delivery of a newly fabricated denture. The patient reported an improvement in overall mastication, deglutition, phonetics, and aesthetics(Figure 10).

\section{Discussion}

The common presentation of Mucormycosis in diabetic individuals is of rhino-orbital-cerebral type. ${ }^{19,20}$ The susceptibility link of diabetes to SARS-CoV-2 infection is unconfirmed; however, disease severity is greatly affected owing to high
A

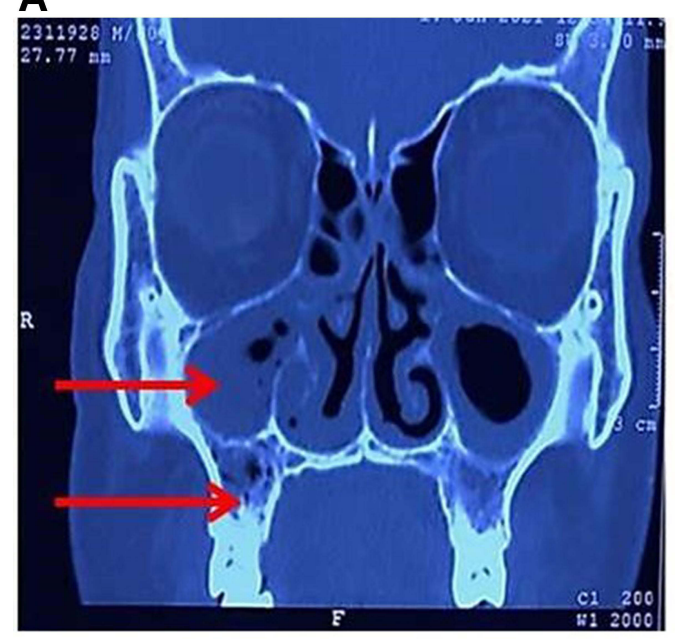

B

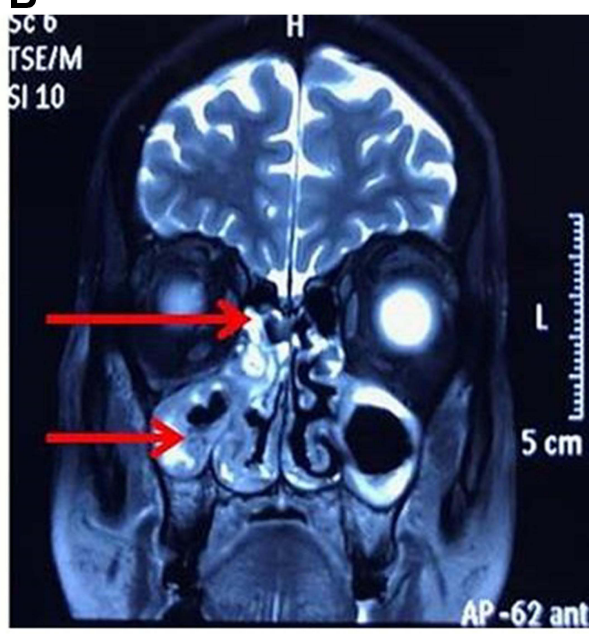

Figure 6 Radiographic investigations showing the extent of the lesion. (A) Plain CT Scan showing frontal, bilateral maxillary and ethmoidal sinusitis with near total opacification of the right maxillary sinus with erosions in the floor and rarefaction of adjacent maxillary alveolus. (B) 3T MRI of brain, orbits and PNS showing diffuse mucosal thickening in the bilateral maxillary and ethmoid sinuses with near total opacification of the right maxillary sinus. 


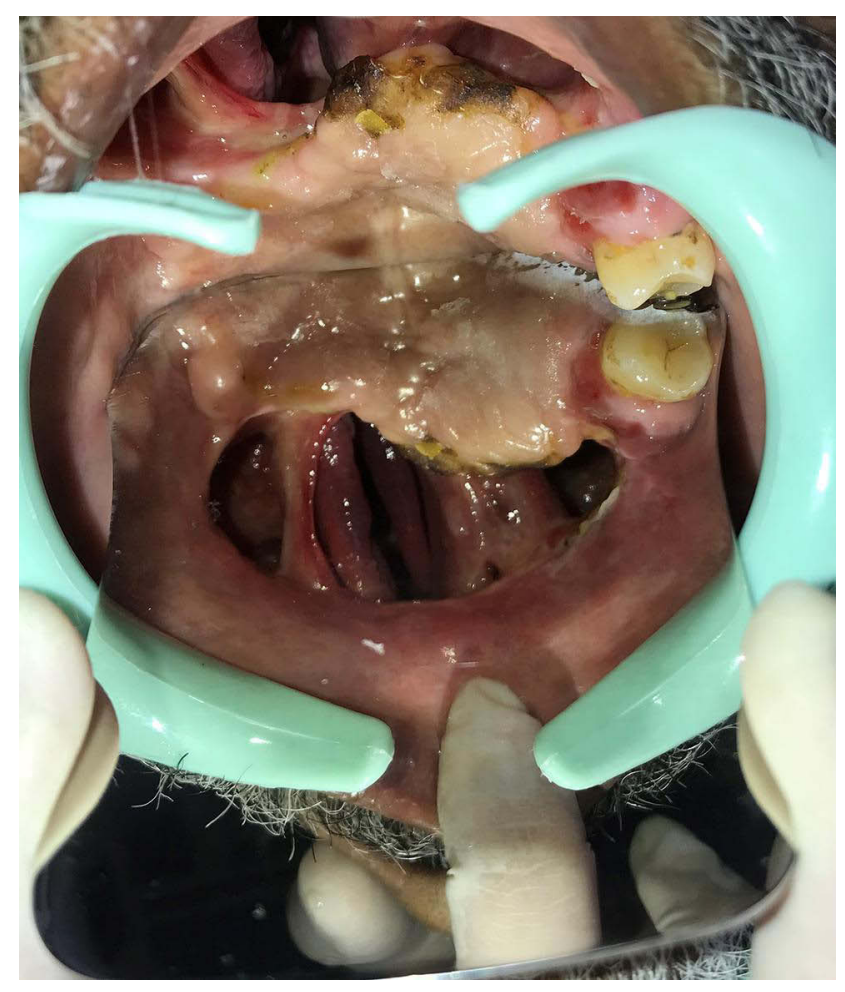

Figure 7 Intraoral maxillary gaping defect with 27 as the only abutment and mobile palatal soft tissue flap.

blood glucose levels, diabetic ketoacidosis, and diabetesassociated endothelial dysfunction. ${ }^{21,22}$ The causative organism Mucorales invade the vessel walls causing thrombosis, ischemia, and necrosis and/or spreads directly through the paranasal sinuses. In a healthy individual, the immune system wards off the infection. However, in an immunocompromised individual, the hyphae forms of Mucorales proliferate easily presenting as a vasotropic fulminant infection. ${ }^{23}$
COVID-19 virus is a multiorgan infection that essentially binds to the Angiotensin Converting Enzyme 2 Receptor and down regulates its activity, leading to an increase in the inflammatory response. Elevated blood sugar also has an exaggerated inflammatory response like in COVID-19. ${ }^{24}$ Viral destruction of immune cells and endothelial destruction associated with diabetes lead to the presentation of cytokine storm causing COVID-associated hypersensitivity reaction. The management of COVIDassociated hypersensitivity response is done with the use of corticosteroids. The use of corticosteroids as a lone entity cannot cause mucor but in combination with diabetes and COVID-19 gives a dreadful fatal infection of Mucormycosis $^{1,25,26}$ The case series presents 2 cases with a common history of uncontrolled diabetes and COVID-19 infection that warranted extensive surgical resection of the maxilla, alveolar process, teeth, soft tissue along with complications like microstomia making the outcome of prosthetic rehabilitation unpredictable and the prognosis poor.

Prosthetic rehabilitation using an obturator prosthesis separates the communication between the oral cavity and nasal cavity acquired after surgical resection. Surgical resection alters facial appearances, imposes psychological and social disability, hampers functions like mastication, swallowing, and speech, however, a carefully designed obturator prosthesis improves masticatory efficiency, speech intelligibility, and relieves psychological distress. ${ }^{27,28}$

Such extensive defects are best rehabilitated by an obturator retained by zygomaticus and nasal implants. However, the presence of absolute contraindications like an underlying systemic immunocompromise, a small volume of
A

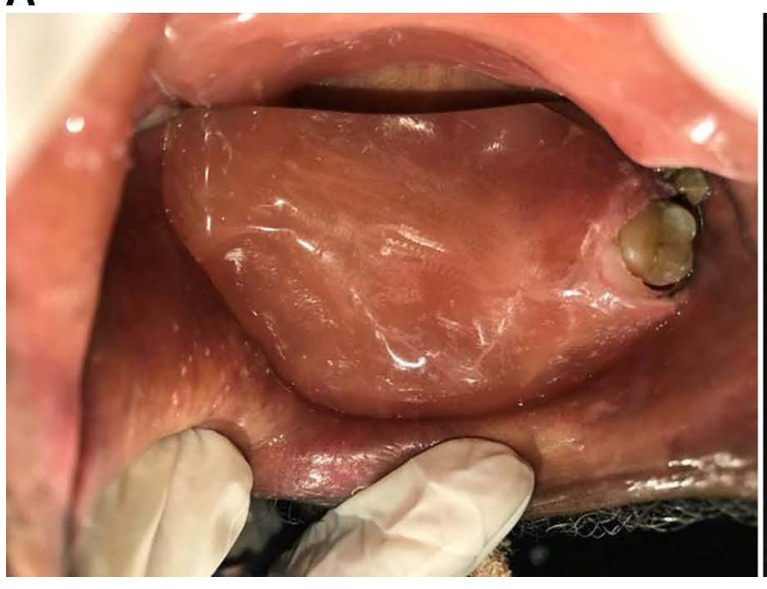

B

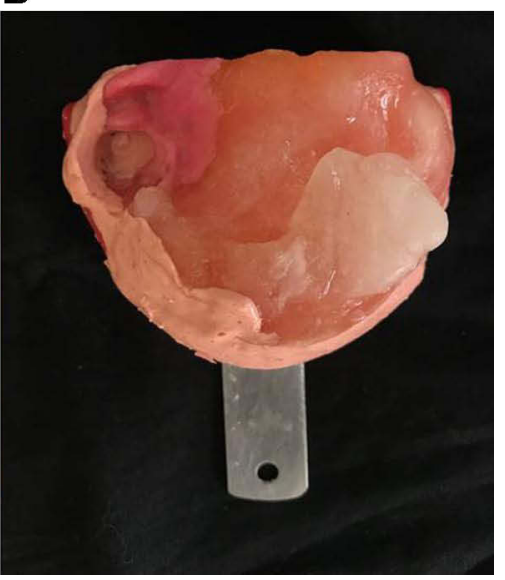

Figure 8 Modification of Conventional Dynamic Impression Technique for accuracy and maximum soft tissue support. (A) Heat cured transitional plate fabricated to record the peripheral seal. (B) The transitional plate coated with acrylic tissue conditioner, worn for the next 24 hours and a pick up impression made. 

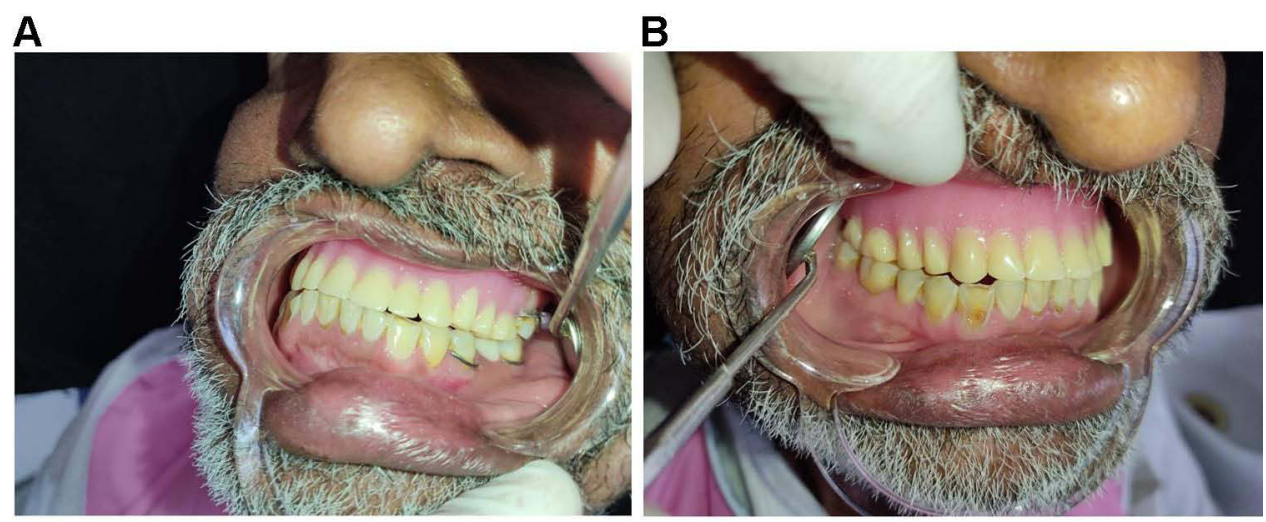

C

D
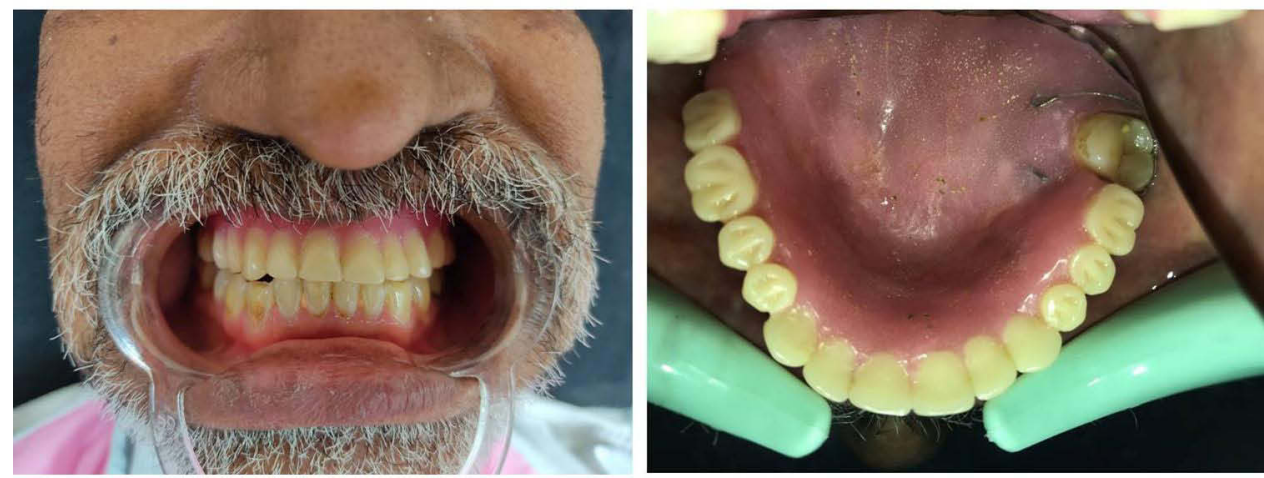

Figure 9 Intraoral view of the obturator prosthesis. (A) Left lateral, (B) right lateral, and (C) frontal views of the occlusion of the monoplane teeth opposing natural teeth. (D) Occlusal view showing zero degree teeth for reduction of lateral force on the abutment tooth and residual ridge.

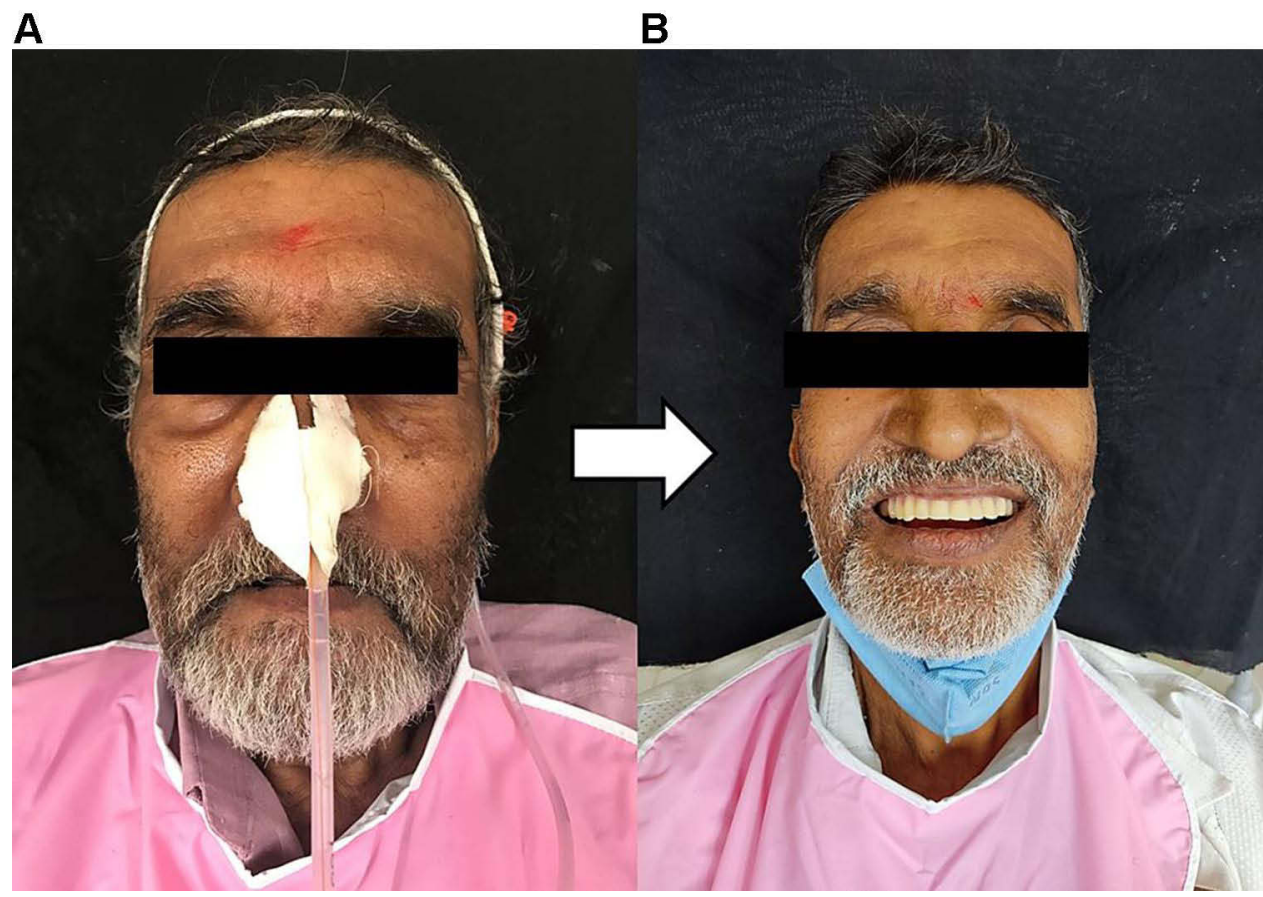

Figure 10 Extraoral view (A) pre-operative extraoral photograph with the Ryle's tube in place. (B) Post-operative extraoral photograph with restoration of function, phonetics and aesthetics. 
residual bone in both cases made the outcome biomechanically dubious. Additionally, the treatment expense could pose a burden for the patients. Hence, modifications were employed in the conventional approach, for the fabrication of the two obturators suiting individual case requirements.

An accurate impression of the basal tissues and/or adjacent teeth is the first stepping stone to making a denture with good retention, stability, and support. There is a need for modification of impression making in both the cases that have been described in this article.

After surgical resection, loss of soft and hard tissue accompanied with microstomia as a post-operative complication further increases the complexity of the case owing to the extent of the defect and reduced mouth opening. The use of a sectional tray and sectional prosthesis has been suggested to be useful in such cases for the ease of impression making and prosthesis insertion. ${ }^{29}$ The impression made in the first case was done in part to record the extent of the defect using the impression compound and reoriented with the pickup impression made using irreversible hydrocolloid impression material and heavy body elastomeric impression material. $^{30}$ The sections of the prosthesis were reapproximated by the use of these intraoral magnets to increase patient comfort. The use of neodymium-iron-boron was done as they have extremely high magnetic saturation and good resistance to demagnetization. ${ }^{31}$

A major complication of the lack of almost the entire basal seat, the presence of only one adjacent tooth, and a mobile soft tissue flap was observed in the second case. A heat-cured palatal plate was fabricated which was followed by the application of a tissue conditioner on the intaglio surface. It gave 2 main advantages first was capturing the functional impression of the defect and the accurate reproduction of the anatomic extent and undercuts utilizing the property of extended flow of the tissue conditioner. Additionally, during these 24 hours, the viscoelasticity of the material maintained a cushioning effect between the tissues lining the defect and the plate. ${ }^{32}$ According to the dynamic impression technique by Chase and Tryde, the final pickup impression, taken after 24 hours, had given a positive replica, as nearly accurate as possible to the defect. $^{33}$ Both the obturators were made hollow using the lost salt technique to improve the ease and comfort of the patient by reducing the weight of the prostheses.

There was an evident gradual postoperative improvement in their masticatory efficiency, swallowing capacity, and speech intelligibility over 24 hours, 48 hours, 72 hours, and 1 week. Both the patients had reported a refinement in the pronunciation and enunciation of syllables, spoken in the local vernacular language. This brought about a definite reduction in the psychological and physical trauma, with the ultimate improvement in the general health of the patients.

Also, neither of the patients had been fully vaccinated against the COVID-19 disease. Hence, the scope of further research in this aspect can be assigned to establishing a definitive positive correlation between complete vaccination and a reduced prevalence of Mucormycosis with more evidence and literature. Furthermore, the use of magnets leaves another open field for further evidence of the durability of the magnets, for predicting the longevity of the prosthesis, if used in similar cases.

\section{Conclusion}

The highly invasive pathogenesis of Mucormycosis often requires an extensive surgical resection that can not only cause facial deformation but also majorly hamper mastication, deglutition, phonetics, etc. This causes deterioration in the quality of general and oral health. It becomes imperative to rehabilitate these patients for a reversal of these effects at the earliest with the help of obturators, the fabrication of which can be extremely tricky.

\section{Future Scope/Clinical Significance}

The two techniques employed in this case series are unique, just like the nature of each case. The modified techniques employed give an added advantage to the overall improvement in function and aesthetics by enhancing retention, support, stability, and patient comfort for future treatment procedures to be followed.

\section{Abbreviations}

COVID-19, Corona Virus Disease of 2019; SARS-CoV2, Severe Acute Respiratory Syndrome CoronaVirus 2; PHEIC, Public Health Emergency of International Concern; WHO, World Health Organization; ROCM, Rhino-Orbito-Cerebral Mucormycosis; FBS, Fasting Blood Sugar; PPS, Postprandial Sugar; HbA1c, Glycated Hemoglobin; MRI, Magnetic Resonance Imaging; CT, Computerized Tomography; PNS, Paranasal Sinuses; TMJ, Temporo Mandibular Joint; RPD, Removable Partial Dentures.

\section{Statement of Informed Consent and Institutional Approval}

Written informed consent was obtained from both the patients for publication of this case report and 
accompanying images. No institutional approval was required to publish the case details.

\section{Disclosure}

The authors report no conflicts of interest in this work.

\section{References}

1. Singh AK, Singh R, Joshi SR, Misra A. Mucormycosis in COVID-19: a systematic review of cases reported worldwide and in India. Diabetes Metab Syndr. 2021;15(4):102146. doi:10.1016/j. dsx.2021.05.019

2. Jeong W, Keighley C, Wolfe R, et al. The epidemiology and clinical manifestations of mucormycosis: a systematic review and meta-analysis of case reports. Clin Microbiol Infect. 2019;25 (1):26-34. doi:10.1016/j.cmi.2018.07.011

3. Prakash H, Ghosh AK, Rudramurthy SM, et al. A prospective multicenter study on mucormycosis in India: epidemiology, diagnosis, and treatment. Med Mycol. 2019;57(4):395-402. doi:10.1093/mmy/ myy060

4. Nithyanandam S, Jacob MS, Battu RR, Thomas RK, Correa MA, D'Souza O. Rhino-orbito-cerebral mucormycosis. A retrospective analysis of clinical features and treatment outcomes. Indian J Ophthalmol. 2003;51(3):231-236.

5. Chamilos G, Lewis RE, Kontoyiannis DP. Delaying amphotericin B-based frontline therapy significantly increases mortality among patients with hematologic malignancy who have zygomycosis. Clin Infect Dis. 2008;47(4):503-509. doi:10.1086/590004

6. Al-Tawfiq JA, Alhumaid S, Alshukairi AN, et al. COVID-19 and mucormycosis superinfection: the perfect storm. Infection. 2021;49 (5):833-853. doi:10.1007/s15010-021-01670-1

7. Centers for Disease Control and Prevention. Treatment for Mucormycosis. Available from: https://www.cdc.gov/fungal/dis eases/mucormycosis/treatment.html. Accessed October 26, 2021.

8. Chigurupati R, Aloor N, Salas R, Schmidt BL. Quality of life after maxillectomy and prosthetic obturator rehabilitation. $J$ Oral Maxillofac Surg. 2013;71(8):1471-1478. doi:10.1016/j. joms.2013.02.002

9. Keyf F. Obturator prostheses for hemimaxillectomy patients. $J$ Oral Rehabil. 2001;28(9):821-829. doi:10.1046/j.1365-2842.2001.00754.x

10. Andrades P. Current strategies in reconstruction of maxillectomy defects. Arch Otolaryngol Head Neck Surg. 2011;137(8):806 doi:10.1001/archoto.2011.132

11. Cornely OA, Alastruey-Izquierdo A, Arenz D, et al. Global guideline for the diagnosis and management of mucormycosis: an initiative of the European confederation of medical mycology in cooperation with the Mycoses Study Group education and research consortium. Lancet Infect Dis. 2019;19(12):e405-e421. doi:10.1016/S1473-3099(19)30312-3

12. Gupta AD, Verma A, Islam JI, Agarwal S. Maxillofacial defects and their classification: a review. IJAR. 2016;4(6):109-114. doi:10.21474/ IJAR01/618

13. Soofi ZI, Yazdanie N, Kazmi SMR. Innovative two-part impression technique for an extensive maxillary defect. $J$ Coll Physicians Surg Pak. 2015;25(10):765767-765793. doi:10.2015/JCPSP.765767

14. Tryde G, Olsson K, Jensen SA, Cantor R, Tarsetano JJ, Brill N. Dynamic impression methods. $J$ Prosthet Dent. 1965;15 (6):1023-1034. doi:10.1016/0022-3913(65)90179-4

15. Francis L. Rehabilitation of a patient with facial and palatal defect a case report. J Clin Diagn Res. 2017;11(3):ZD19-ZD20. doi:10.7860/JCDR/2017/25063.9540

16. Tandon A, Pandey L. COVID-19, steroids, and mucormycosis: what an ophthalmologist should know. Indian J Ophthalmol. 2021;69 (7):1970. doi:10.4103/ijo.IJO_1143_21
17. Salehi M, Ahmadikia K, Badali H, Khodavaisy S. Opportunistic fungal infections in the epidemic area of COVID-19: a clinical and diagnostic perspective from Iran. Mycopathologia. 2020;185 (4):607-611. doi:10.1007/s11046-020-00472-7

18. Bhasin AS, Singh V, Mantri SS. Rehabilitation of patient with acquired maxillary defect, using a closed hollow bulb obturator. Indian J Palliat Care. 2011;17(1):70-73. doi:10.4103/09731075.78453

19. John TM, Jacob CN, Kontoyiannis DP. When uncontrolled diabetes mellitus and severe COVID-19 converge: the perfect storm for mucormycosis. JoF. 2021;7(4):298. doi:10.3390/jof7040298

20. Ak AK, Gupta V. Rhino-orbital cerebral mucormycosis. In: StatPearls. StatPearls Publishing; 2021. Available from: http://www. ncbi.nlm.nih.gov/books/NBK557429/. Accessed October 26, 2021.

21. Dalkiz M, Dalkiz A. The effect of immediate obturator reconstruction after radical maxillary resections on speech and other functions. Dent J. 2018;6(3):22. doi:10.3390/dj6030022

22. Kayina CA, Maitra S, Anand RK, Ray BR, Baidya DK, Subramaniam R. SARS-CoV-2 infection presenting with hyperglycemia and ketosis: a case series of three diabetic patients. Indian J Crit Care Med. 2020;24(11):1141-1142. doi:10.5005/jp-journals-1007123595

23. Afroze SN, Korlepara R, Rao GV, Madala J. Mucormycosis in a diabetic patient: a case report with an insight into its pathophysiology. Contemp Clin Dent. 2017;8(4):662-666. doi:10.4103/ccd.ccd_558_17

24. Logette E, Lorin C, Favreau C, et al. A machine-generated view of the role of blood glucose levels in the severity of COVID-19. Front Public Health. 2021;9:695139. doi:10.3389/fpubh.2021.695139

25. Garg D, Muthu V, Sehgal IS, et al. Coronavirus disease (Covid-19) Associated Mucormycosis (CAM): case report and systematic review of literature. Mycopathologia. 2021;186(2):289-298. doi:10.1007/ s11046-021-00528-2

26. Ahmadikia K, Hashemi SJ, Khodavaisy S, et al. The double-edged sword of systemic corticosteroid therapy in viral pneumonia: a case report and comparative review of influenza-associated mucormycosis versus COVID-19 associated mucormycosis. Mycoses. 2021;64 (8):798-808. doi:10.1111/myc.13256

27. Nilanonth S, Shakya P, Chotprasert N, Srithavaj T. Combination prosthetic design providing a superior retention for mid-facial defect rehabilitation: a case report. J Clin Exp Dent. 2017;9(4):e590-e594. doi: $10.4317 /$ jced. 53513

28. Vero N, Mishra N, Singh BP, Singh K, Jurel SK, Kumar V. Assessment of swallowing and masticatory performance in obturator wearers: a clinical study. $J$ Adv Prosthodont. 2015;7(1):8-14. doi:10.4047/jap.2015.7.1.8

29. Kumar KA, Bhat V, Nair KC, Suresh R. Preliminary impression techniques for microstomia patients. J Indian Prosthodont Soc. 2016;16(3):229-233. doi:10.4103/0972-4052.186400

30. Cervino G, Fiorillo L, Herford AS, et al. Alginate materials and dental impression technique: a current state of the art and application to dental practice. Mar Drugs. 2018;17(1):18. doi:10.3390/ md17010018

31. Prasad M, Manoj-Kumar M, Gowri-Sankar S, Chaitanya N, VivekReddy G, Venkatesh N. Clinical evaluation of neodymium-iron-boron (Ne2Fe14B) rare earth magnets in the treatment of mid line diastemas. $J$ Clin Exp Dent. 2016;8(2):e164-e171. doi:10.4317/jced.52352

32. Taguchi N, Murata H, Hamada T, Hong G. Effect of viscoelastic properties of resilient denture liners on pressures under dentures. $J$ Oral Rehabil. 2001;28:1003-1008. doi:10.1111/j.1365-2842.2001.00770.x

33. Jayaraman S, Singh BP, Ramanathan B, Pazhaniappan Pillai M, MacDonald L, Kirubakaran R. Final-impression techniques and materials for making complete and removable partial dentures. Cochrane Database Syst Rev. 2018;2018(4):CD012256. doi:10.1002/14651858. CD012256.pub2 


\section{Publish your work in this journal}

Clinical, Cosmetic and Investigational Dentistry is an international, peer-reviewed, open access, online journal focusing on the latest clinical and experimental research in dentistry with specific emphasis on cosmetic interventions. Innovative developments in dental materials, techniques and devices that improve outcomes and patient satisfaction and preference will be highlighted. The manuscript management system is completely online and includes a very quick and fair peer-review system, which is all easy to use. Visit http://www.dovepress.com/testimonials.php to read real quotes from published authors. 